\title{
Application of portable optical laboratory in high schools and colleges
}

Gregory Altshuler, Nickolai Belashenkov, Vladimir Ermolaev, Mickle Inochkin, Vyatcheslav Karasev

Gregory B. Altshuler, Nickolai R. Belashenkov, Vladimir S. Ermolaev, Mickle V. Inochkin, Vyatcheslav B. Karasev, "Application of portable optical laboratory in high schools and colleges," Proc. SPIE 2525, 1995 International Conference on Education in Optics, (13 October 1995); doi: 10.1117/12.224041

Event: SPIE's 1995 International Symposium on Optical Science, Engineering, and Instrumentation, 1995, San Diego, CA, United States 
Application of portable optical laboratory in high-schools and colleges

Grigori B.Altshuler, Nickolai R.Belashenkov, Vladimir S.Ermolaev, Michael V.Inochkin, Vyacheslav B.Karasev

St.-Petersburg State Institute of Fine Mechanics \& Optics, 14, Sablinskaya st., 197101 St.Petersburg, Russia

\begin{abstract}
The present paper describes the experience of application of portable optical laboratory in optical practicum developed directly for training and demonstrations of basic optical laws and phenomena in high-schools, colleges and non-technical universities all over Russia. The laboratory includes the portable optical platform with built-in laser and lamp sources, kit of optical components and software. These accessories provide the attractive and smart teaching in general optics during lectures, lessons and practice at schools and colleges. The portable optical laboratory provides 28 basic lab works and demonstrations in reflection, refraction, absorption and dispersion of light, interference, diffraction, polarization of light, image formation and waveguide propagation of light in optical fibers. Due to their interdependence one can teach and learn a whole course of general optics. The individual work of students and school children with optical kit stimulates and develops their creative abilities and experimental skills, as well increases the effectiveness of education. The kit is provided with optional elements for a number of extra experiments with holography, polarizing light propagation, simple optical devices etc. These extensions allow to modify the education process according to teacher's point of view. The conception of optical class-room based on portable optical laboratories is discussed. The effectiveness of individual and small-group training is analyzed.
\end{abstract}

\title{
1. INTRODUCTION
}

The optics is one of the few sciences which fundamental principles may be illustrated by simple and available demonstrations and experiments providing the comprehensive teaching and learning. It is not necessary to prove the importance of practical training in physical education. It is essentially important for mechanics and optics where the most phenomena and laws could be easily demonstrated. Practical training in physics and particularly in optics becomes more important also because of worring tendency of last years: computer modelling substitutes the "live" experiment so students get the aberrated conception of real physics.

Recently the new portable optical laboratory (POL) named "OPTIK" has been developed in Saint-Petersburg Institute of Fine Mechanics \& Optics. This project had been launched 6 years ago and many specialists from different schools, colleges and universities all over Russia participated in this project at different stages of its realization. Now the development is completed. More than 100 POLs are installed in different cities of Russia and abroad and being used for optical training.

In present paper we describe the main features of POL, summarize the experience of its application and analyse the advantage and possible ways of development of optical practicum based on "OPTIK" accessories. We represent the methods of training in optics as well the list of demonstrations and lab works which could be realised using POL's facilities.

\section{TYPES OF TRAINING OPTICAL LABORATORIES}

In general there are three main types of optical laboratories which are applied now in optical practicum (see fig.1). The simplest kits which are most effective at initial stages of training are applied as usual in secondary and high schools and partially in colleges ${ }^{1}$. The universal training laboratories provide training in different optical phenomena ${ }^{2}$. These laboratories are widely used in high schools, colleges, short courses and even in universities. Third 
type of optical training laboratories are used mainly in universities as they are specialized laboratories which practically are used both in optical training and scientific research ${ }^{3}$.

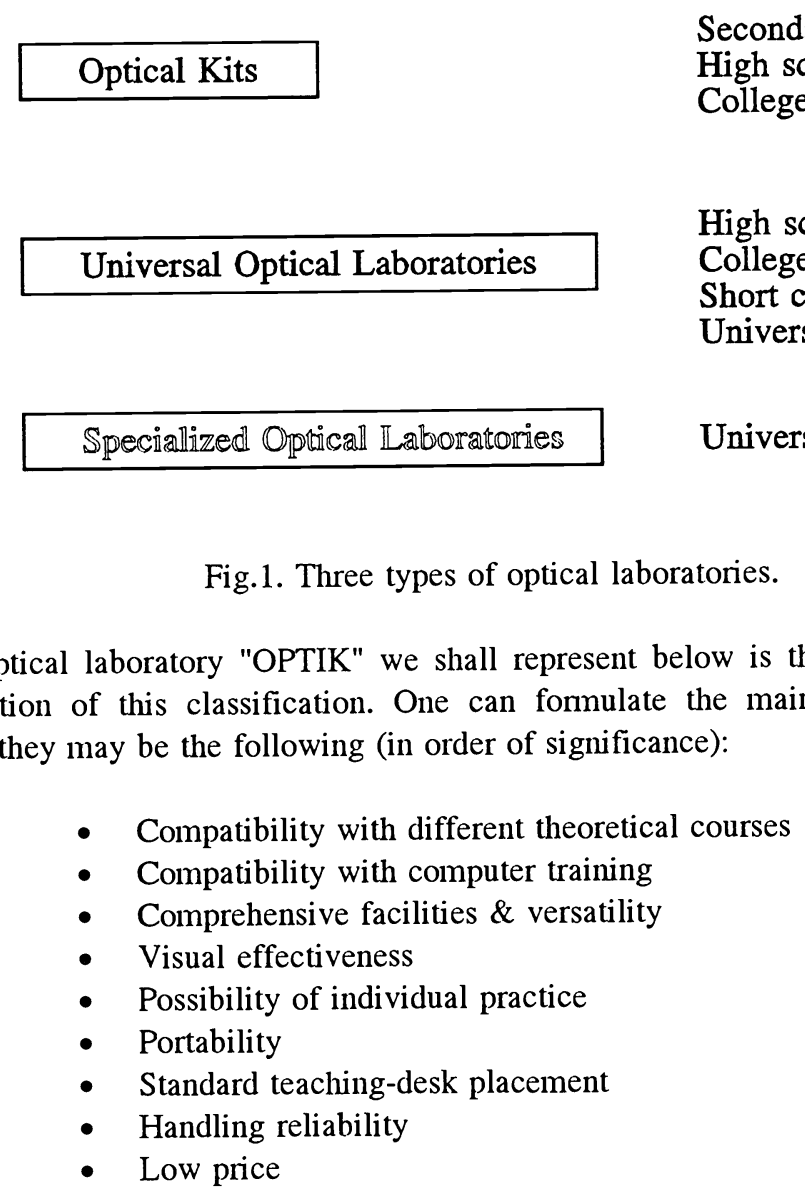

The portable optical laboratory "OPTIK" we shall represent below is the universal training laboratory so it belongs to second position of this classification. One can formulate the main requirements to universal training laboratory. In our mind they may be the following (in order of significance):

- Compatibility with different theoretical courses

- Compatibility with computer training

- Comprehensive facilities \& versatility

- Visual effectiveness

- Possibility of individual practice

- Portability

- Standard teaching-desk placement

- Low price

The universal optical training laboratory must be compatible with different theoretical courses and so it has to provide a large number of lab experiments. It is necessary that physical laboratory has to be compatible with computer means of training commonly used at different stages of education. It should have high demonstration effectiveness to attract and excite students for optics. Students should be able to make most of lab experiments individually. The later requirement means that portable version of optical laboratory is preferable so it could be easily installed on any desk and handling reliability is necessary.

While the analysis of possible design and composition of optical laboratory has been made it was found out that it is not easy to satisfy all mentioned requirements in one device. However it seems that we have made it in POL "OPTIK".

\section{PORTABLE OPTICAL LABORATORY "OPTIK": COMPOSITION, TUTORIAL \& APPLICATION}

The portable optical laboratory "OPTIK" includes the appliance "OPTIK", training software for parallel computer modelling and tutorial instructions. So it could easily realise one of the most convenient method of optical education combining the theoretical course (lectural demonstration of different optical phenomena are available), computer simulation of optical effects and knowledge testing via comprehensive software and individual or smallgroup practical work with real optical components. One of the possible application of POL "OPTIK" in physical classroom is illustrated in fig.2. 


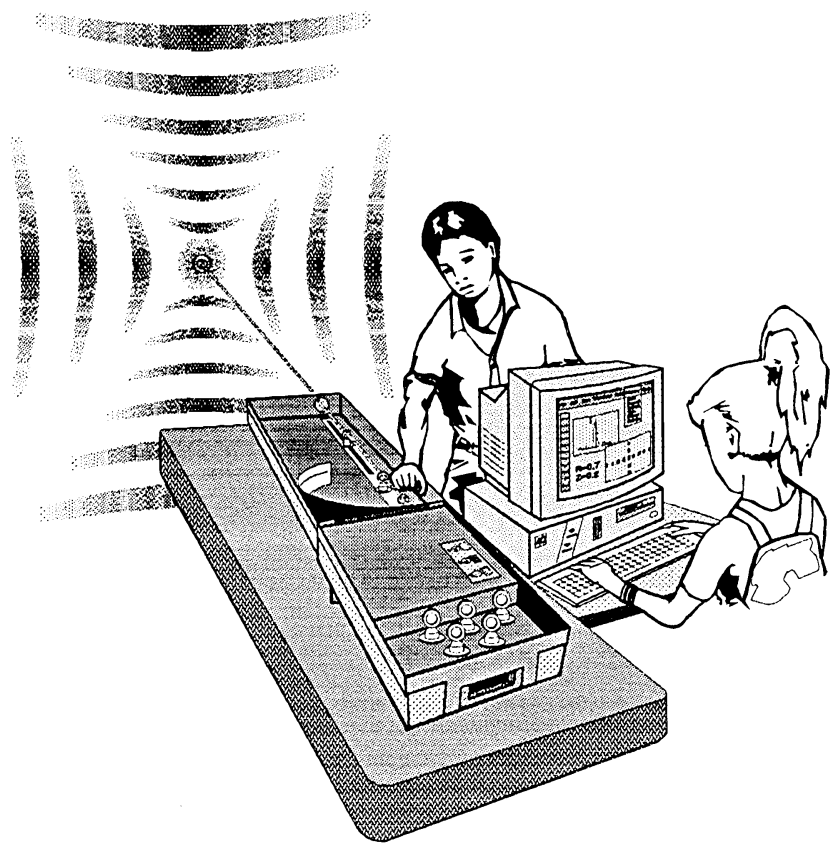

Fig.2. The general view of possible POL "OPTIK" application in physical classroom.

The appliance "OPTIK" has a case design. The foldable optical platform has a small size and provides portability and low weight of the appliance reserving the necessary rigidity of construction adopted in optics. The general top view of optical platform is represented in fig.3.

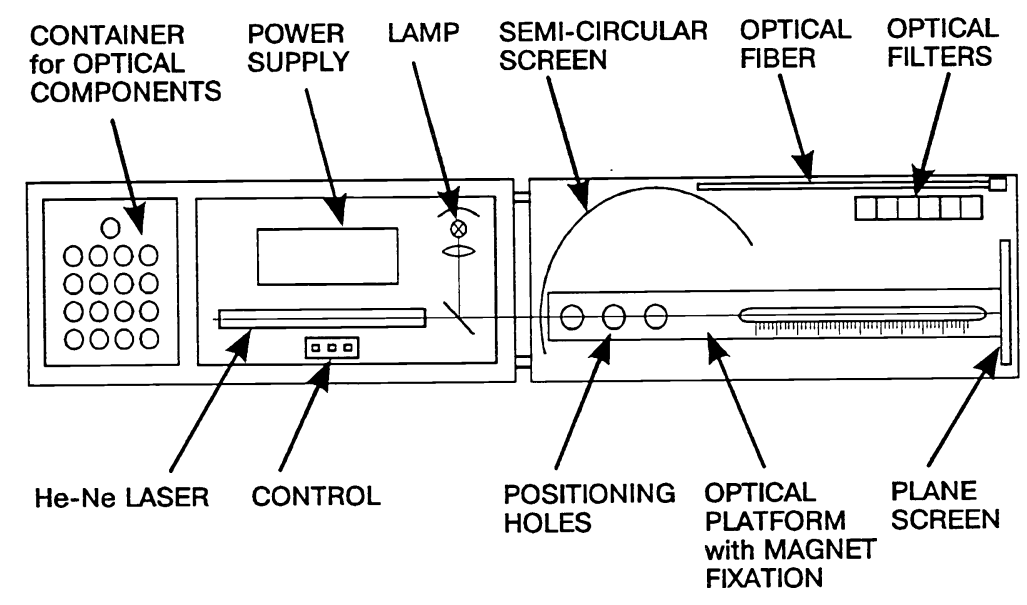

Fig.3. The top view of "OPTIK" appliance optical platform.

The optical platform contains the built-in small light sources (He-Ne laser, lamp). The application of coherent and incoherent light sources combination provides the possibility of an original teaching technique in modern optics. The wide set of optical components mounted in unified holders allows instructor to include the understandable and attractive optical demonstrations in lectures and practice. 
Two part of case being unfolded and installed (see, for example, fig.2) are positioned with high accuracy. The later feature as well as the design of optical holders with magnet fixation allow to construct optical schemes and demonstrate optical phenomena using available optical components without prealignment. Thus the performance of any available experiment takes less than a half of minute that is very important especially during lectural demonstration. The appliance "OPTIK" provides 28 lab experiments. All these experiments can be carried out individually. Most of them instructor can demonstrate on big screen. Thus "OPTIK" may be used as desk-top device as well as the device for lectural demonstrations.

The list of 28 available lab works and demonstration is subdivided in 5 groups represented below.

\section{INTRODUCTION IN OPTICS}

- $\quad$ "Properties of Light. The comparison of lamp and laser radiation"

- "Main optical phenomena and laws"

- "Optical Components"

\section{GEOMETRICAL OPTICS}

- "Reflection of Light"

- "Refraction of Light"

- "Ray Trace in a Wedge"

- "Ray Trace in a Prism"

- "Ray Trace in a Flat Plate"

- "Total Internal Reflection of Light"

- "Optical Fibers"

- "Dispersion of Light"

- "Positive Lens"

- "Image Formation"

- "Negative Lens"

- "Plane Waves"

\section{WAVE OPTICS}

- "Polarization of Light"

- "Polarization of Light under Reflection"

- "Interference of Light"

- "Diffraction of Light on a Slit"

- "Diffraction of Light on Rectangular and Triangular Apertures"

- "Diffraction of Light on a Circular Aperture"

- "Diffraction of Light on Two Apertures (Young's Experiment)

- "Holography"

- "Scattering of Light" 


\section{MEASUREMENTS}

- "Measurement of Focal Distance of a Positive Lens"

- "Measurement of Refraction Index"

- $\quad$ Measurement of Refraction Index via the Minimum Light Deflection Angle in a Prism"

- $\quad$ "Measurement of Refraction Index via the Ray Trace in a Wedge"

- $\quad$ Measurement of Refraction Index via the Extremum Angle of Total Internal Reflection of Light"

- "Measurement of Refraction Index via the Whole Refraction Cone"

- "Measurement of Light Wavelength Using Fraunhofer Diffraction"

\section{OPTICAL APPLICATIONS}

- "Telescope"

- "Spectroscope"

- "Microscope"

- "Optical communication"

- "Optical recording"

- "Optical processing of information"

- "Optics of eye"

- "Optics of tooth"

The last group of experiments is very important because it helps to describe the role of optics in various life applications.

\section{CONCLUSIONS}

The portable optical laboratory "OPTIK" represented and described above seems to be very useful and convenient tool in optical education. It may be applied to general optics course in high schools, colleges and universities. It may be used both for lectural demonstrations and individual work of students.

\section{REFERENCES}

1. D.C.O'Shea "The OSA Optics Discovery Kit - its development and use", Proc. SPIE, Education in Optics, v.1603, pp.368-377, 1991

2. S.F.Mironov, A.A.Stolov, A.I.Fishman "Universal laboratory complete for optical practice", Proc. SPIE, Education in Optics, v.1603, pp.523-526, 1991

3. G.B.Altshuler, N.R.Belashenkov, A.V.Okishev "New Approach in Laser Physics Education", Proc. SPIE, Education in Optics, v.1603, pp.332-337, 1991 